\title{
Collinearity Problems in Passive Target Localization Using Direction Finding Sensors
}

\author{
Barış Fidan ${ }^{\# 1}$, Samuel P. Drake ${ }^{* 2}$, Brian D. O. Anderson ${ }^{\# 3}$, Guoqiang Mao ${ }^{\S 4}$, Anushiya A. Kannan ${ }^{\S 5}$ \\ \# National ICT Australia (NICTA) and \\ Research School of Information Sciences and Engineering, Australian National University, Canberra, Australia \\ 1 Baris.Fidan@nicta.com.au, 3 Brian. Anderson@nicta.com.au \\ * Defence Science and Technology Organisation (DSTO), Edinburgh, Australia \\ ${ }^{2}$ Sam.Drakeddsto.defence.gov.au \\ $\S$ National ICT Australia (NICTA) and \\ School of Electrical and Information Engineering, University of Sydney, Australia \\ 4 Guoqiang.Mao@nicta.com.au, ${ }^{5}$ Anushiya.Kannan@nicta.com.au
}

\begin{abstract}
In passive target localization using direction finding (DF), there are particular sensor-target placements that cause large biases in the estimates or the failure of estimates to converge to a unique solution. Identification of such problematic configurations is crucial for implementing estimation and tracking algorithms effectively. In this paper we propose four methods for characterizing near-collinearity problems in a sensor-target configuration which enable one to quickly and easily identify cases in which DF-based localization will fail to obtain a solution or give unreliable results.
\end{abstract}

\section{INTRODUCTION}

Passive localization of targets has a wide variety of applications ranging from navigation to robotics [1]-[4]. The advantage of passive localization techniques over active ones such as radar and sonar is that they use much less energy and the activity is more easily concealed. The disadvantage of passive localization is that it often requires the target to be an emitter ${ }^{1}$ transmitting some signal and, depending on the technique used, some information about this signal may be required. There are a number of passive localization techniques including those based on time difference of arrival (TDoA), differential doppler, and direction finding (DF); these algorithms are summarized in a defence context in [1], [5]. In this paper we focus on DF localization techniques. DF is the process whereby the direction of an incoming electromagnetic signal is determined. DF localization is also known as angle of arrival (AoA), direction of arrival (DoA) and line of bearing (LoB) localization. One of the advantages of using DF sensors is that they do not require a priori knowledge of the signal or any particular feature of the signal.

Passive localization with DF sensors can be done in two or three dimensions, the latter requiring both azimuth and elevation. In this paper we consider only two dimensional localization, assuming that the emitter is located on the ground. Localization of emitters that are known to be on the surface of the Earth is often referred to as geolocation, noting that

\footnotetext{
${ }^{1}$ In this paper, we assume that the target is an emitter and use the term "emitter" in place of "target" as well.
}

the word "geolocation" does not imply that the sensors are also ground-based. If a DF sensor is not ground-based, or if the emitter does not lie on the horizontal plane of the reference frame of this DF sensor, then the azimuth angle measured by the sensor will not be the actual azimuth angle even in the absence of noise. The error between the actual and measured azimuths depends on the elevation angle as well as the measurement noises [1]. In such cases, we assume that the elevation angle affect on azimuth measurement is eliminated.

The seminal works on algorithms and error estimates for DF localization were done in the 1940's and 1950's [2], [6], [7]. A review of DF and other passive localization techniques can be found in [3]. The first and second moments of noisy DF measurements are the bias and the covariance. Both the Stansfield algorithm and the maximum likelihood estimator (MLE) [8] produce a bias in the emitter location estimate, although the bias in MLE approaches zero as the number of measurements approaches infinity [4]. In both cases the magnitude and direction of the bias is sensitive to the DF sensor and emitter locations. A comprehensive study of bias in linear least square algorithms for DF localization is found in [9], in which the bias for a linear array of sensors is shown to decrease as the range to baseline ratio decreases.

Components of the covariance matrix also depend on the DF localization algorithm. However for unbiased estimators the error covariance matrix is lower bounded by the inverse of the Fisher information matrix (FIM), which is the CramerRao lower bound [10]. The area of the covariance ellipse [10] is proportional to the determinant of the covariance matrix and for efficient estimators inversely proportional to the determinant of the FIM. The optimal sensor placement for passive localization is found by minimizing the area enclosed by the covariance ellipse which is equivalent to minimizing the determinant of the covariance matrix or maximizing the determinant of the FIM.

The FIM determinant has also been used to analyze the optimal sensor positions for static bearing-only localization in [11], [12] as well as investigating the optimal receiver 
trajectories for a single sensor and a single emitter in [13]. [13] notices, based on simulation results, that if the effective baseline-to-range ratio is small, then maximizing the bearing rate is of primary importance, being equivalent to the requirement of reducing the collinearity measures (as described later).

In this paper, unlike the previously mentioned approaches, rather than identifying optimal sensor arrangements, we examine some measures that identify when a particular arrangement of sensors and the emitter are "problematic". By "problematic" we mean that bearings lines do not intersect and therefore there is no solution, or that the solution is much further from the true emitter location than can be expected from the error covariance matrix, or the covariance matrix is too large. We show that problematic solutions can be identified by collinearity measures and we establish four different measures.

\section{Geolocation Using Direction Finding Sensors}

A typical DF-based geolocation algorithm to locate the position of an emitter $E$ on a 2-dimensional $x y$-plane relies on fusion of direction measurements taken by a set of DFsensors $\left\{S_{1}, \ldots, S_{n}\right\}^{2}$. Ideally, assuming that the direction measurements are perfect, the direction measurement of each sensor $S_{i}$ correspond to the ray $\overrightarrow{S_{i} E}$ originating from $S_{i}$.

In a realistic case, even if the position of each sensor $S_{i}$ is known accurately, one needs to allow for a direction measurement error $\tilde{\theta}_{i}=\hat{\theta}_{i}-\theta_{i}$ between the actual bearing $\theta_{i}$ (defined above) and the measured bearing $\hat{\theta}_{i}$, where $\theta_{i}, \hat{\theta}_{i} \in\left(-180^{\circ}, 180^{\circ}\right]$.

Assumption 1: For the sensors $S_{1}, \ldots, S_{n}$, the bearing errors $\tilde{\theta}_{1}, \ldots, \tilde{\theta}_{n}$ are modelled as independent and identically distributed (i.i.d.) Gaussian variables with zero-mean and variance $\sigma_{i}$, where the value of $\sigma_{i}$ depends on the properties of the sensor $S_{i}$ and the environment as well as certain characteristics of the emitter $E$ such as its lateral beam width. ${ }^{3}$

Using the above Gaussian model, in order to indicate high-probability location regions for the emitter, for each sensor-measurement pair $\left(S_{i}, \hat{\theta}_{i}\right)$, one may use the $\sigma$-cone $C_{\sigma}\left(S_{i}, \hat{\theta}_{i}\right)$ via the conic region between the two rays originating from $S_{i}$, one having counter-clockwise angle $\hat{\theta}_{i}-\sigma$ and the other $\hat{\theta}_{i}+\sigma$, the $2 \sigma$-cone $C_{2 \sigma}\left(S_{i}, \hat{\theta}_{i}\right)$, the $3 \sigma$-cone $C_{3 \sigma}\left(S_{i}, \hat{\theta}_{i}\right)$, etc. Note that, given the measurement $\hat{\theta}_{i}$, the probabilities that emitter $E$ lies in $C_{\sigma}\left(S_{i}, \hat{\theta}_{i}\right), C_{2 \sigma}\left(S_{i}, \hat{\theta}_{i}\right)$, $C_{3 \sigma}\left(S_{i}, \hat{\theta}_{i}\right)$ are $68.3 \%, 95.5 \%, 99.7 \%$, respectively. Similarly, given the location of $E$, i.e., given the actual bearing $\theta_{i}, C_{\sigma}\left(S_{i}, \theta_{i}\right), C_{2 \sigma}\left(S_{i}, \theta_{i}\right), C_{3 \sigma}\left(S_{i}, \theta_{i}\right), \ldots$ can be used to indicate the high-probability regions for the location estimate

\footnotetext{
${ }^{2}$ In the sequel we abuse the notations $E$ and $\left\{S_{1}, \ldots, S_{n}\right\}$ to denote, respectively, the emitter and the sensors themselves as well as their positions on the $x y$-plane. Hence the expression $S_{i}=\left[x_{i}, y_{i}\right]^{T}$ indicates that the $x$ and $y$ coordinates of $S_{i}$ are, respectively, $x_{i}$ and $y_{i}$.

${ }^{3}$ Although a Gaussian variable can assume values on $(-\infty, \infty)$ while an angle is limited to an interval of length 360 (degrees), so that the assumption might not appear to be appropriate, $\sigma$ is sufficiently small in practice that the apparent contradiction is immaterial.
}

$\hat{E}$ that would be obtained using the bearing measurement $\hat{\theta}_{i}$. Consider a given fixed geometric setting of $n(\geq 2)$ sensors one emitter, $\left\{S_{1}, \ldots, S_{n}\right\}$-E. We call

$$
R_{e \sigma} \equiv R_{e \sigma}\left(S_{1}, \ldots, S_{n}, E\right) \triangleq \bigcap_{i=1}^{n} C_{\sigma}\left(S_{i}, \theta_{i}\right)
$$

the expected detection uncertainty region (EDUR) of emitter $E$ with respect to sensors $S_{1}, \ldots, S_{n}$.

\section{Collinearity Problems in Geolocation with TWO SENSORS}

\section{A. Defining and Measuring Collinearity}

As already noted, a particular geometric problem in cooperative emitter localization is collinearity, which may result when the emitter and the DF sensors are exactly or nearly collinear. The notions of exact and near collinearity are depicted in Fig. 1. Note that in the case of an exact collinearity and noiseless measurements the emitter can not be located uniquely using only the directions from two sensors. Similarly in a near-collinearity case, a small error in one or both of the direction measurements may cause non-unique localization of the emitter $E$, while if there is an intersection, its location may be extremely sensitive to changes in measurement errors.

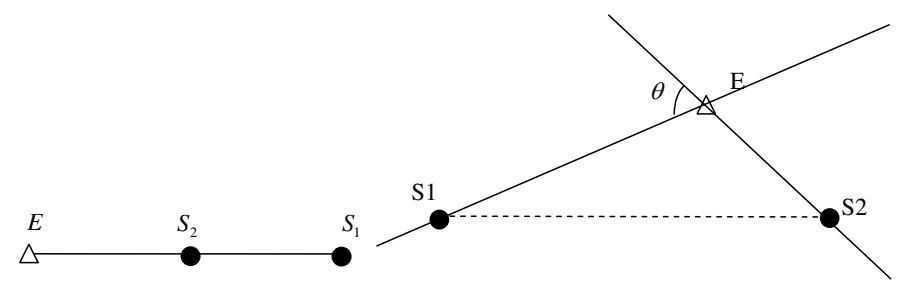

(a)

(b)

Fig. 1. (a) Exact collinearity: $E, S_{1}, S_{2}$ are collinear. (b) Near collinearity occurs when $\theta$ is close to $0^{\circ}$ or $180^{\circ}$.

To define near collinearities formally with an ultimate aim of determining the emitter positions in a given area for a given set of sensors (with positions) for which a collinearity problem is likely to occur, one needs to define a collinearity measure (CM) first. Once the $\mathrm{CM}$ is defined and a threshold for being collinearity-problem-free is fixed, the problematic emitter locations in the region of interest can be determined.

For a two sensors - one emitter setting, such as the one depicted in Fig. 1(b), this measure may be based on the cones $C_{\sigma}\left(S_{1}, \theta_{1}\right), C_{\sigma}\left(S_{2}, \theta_{2}\right)$ and the EDUR $R_{e \sigma}\left(S_{1}, S_{2}, E\right)$ or the subtended angles of the triangle $E S_{1} S_{2}$. In the next subsections, we consider both of these two approaches.

\section{B. A Measure Based on Detection Uncertainty Regions}

Considering the direction measurement uncertainties, we define a CM for the two sensors - one emitter setting depicted in Fig. 1(b) based on the EDUR $R_{e \sigma}\left(S_{1}, S_{2}, E\right)$ by

$$
C M_{1}\left(S_{1}, S_{2}, E\right) \triangleq \operatorname{Area}\left(R_{e \sigma}\left(S_{1}, S_{2}, E\right)\right),
$$


Note that it is also possible to define the CM using a lineal measure (e.g., the diameter) of $R_{e \sigma}$. The larger the measure of $R_{e \sigma}$, the larger the uncertainty and the extent of the collinearity problem will be. Example cases are illustrated in Fig. 2. Fig. 3(a) depicts the problematic region

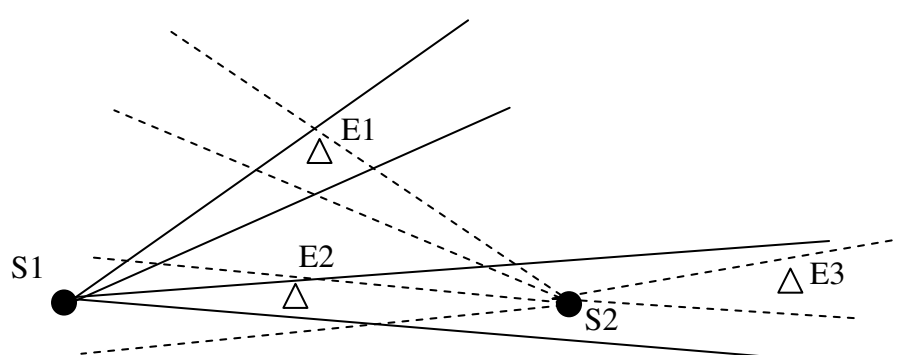

Fig. 2. Measuring collinearity based on EDURs: $E_{1}$ does not suffer from a collinearity problem but $E_{2}$ and $E_{3}$ do. For each of $E_{1}, E_{2}, E_{3}$, the solid lines indicate the borders of the $\sigma$-cone $C_{\sigma}\left(S_{1}, \theta_{1}\right)$ and the dashed lines indicate the borders of the $\sigma$-cone $C_{\sigma}\left(S_{2}, \theta_{2}\right)$.

$\left\{E \quad: C M_{1}\left(S_{1}, S_{2}, E\right) \geq \overline{C M}_{1}\right\}$ for $S_{1}=[0,0]^{T}(\mathrm{~km})$, $S_{2}=[1,0]^{T}(\mathrm{~km}), \sigma=5^{\circ}$ and threshold $\overline{C M}_{1}=0.03\left(\mathrm{~km}^{2}\right)$.

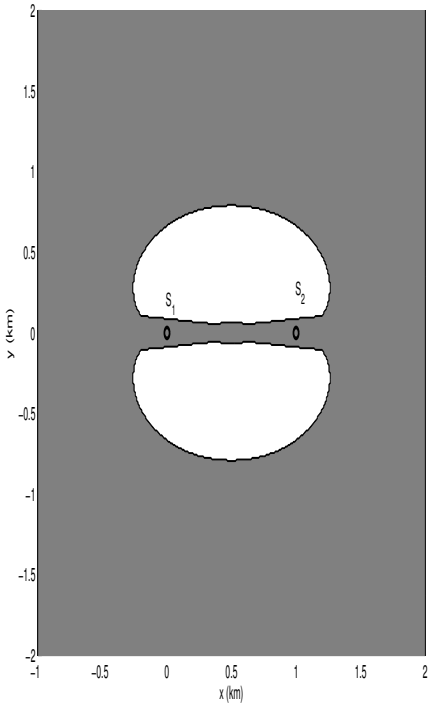

(a)

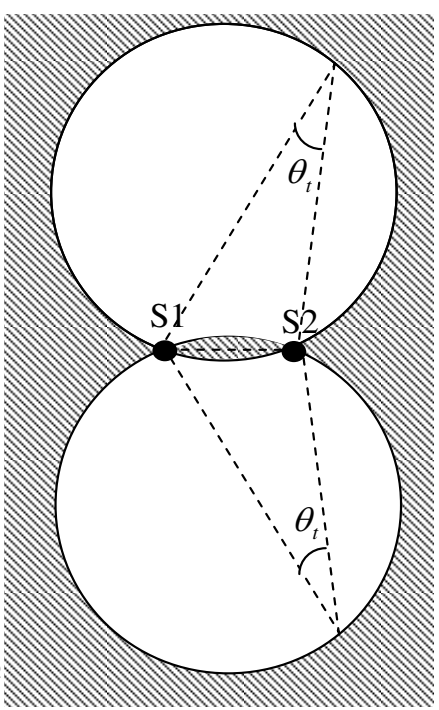

(b)
Fig. 3. Problematic regions (a) $\left\{E\right.$ : $\left.C M_{1}\left(S_{1}, S_{2}, E\right) \geq \overline{C M}_{1}\right\}$ with $\sigma=5^{\circ}, \overline{C M}_{1}=0.03\left(\mathrm{~km}^{2}\right)$ and (b) $\left\{E: C M_{2}\left(S_{1}, S_{2}, \bar{E}\right) \geq \overline{C M}_{2}=\right.$ $\left.1 / \theta_{t}\right\}$ for $S_{1}=[0,0]^{T}(\mathrm{~km}), S_{2}=[1,0]^{T}(\mathrm{~km})$.

\section{Measures Based on Subtended Angles}

The CM (2) requires calculating the area of a non-regular quadrilateral intersection region in each setting, which brings a level of computational burden, especially while determining the problematic regions in the region of interest. In this subsection, we define three alternative CMs for the two sensors - one emitter setting depicted in Fig. 1(b) based on the subtended angles of the triangle $E S_{1} S_{2}$, which are computationally simpler to implement than (2): A simple single-angle- based CM, a CM based on stochastic properties of the DF measurement errors, and a Jacobian-based CM.

1) A Single-Angle-Based Measure: As a simple CM, we propose the use of the reciprocal of the smaller angle between the lines $S_{1} E$ and $S_{2} E\left(\min \left\{\theta, 180^{\circ}-\theta\right\}\right.$ in Fig. 1(b)):

$$
C M_{2}\left(S_{1}, S_{2}, E\right) \triangleq 1 / \min \left\{\theta, 180^{\circ}-\theta\right\}
$$

The corresponding threshold $\overline{C M}_{2}$ effectively defines the minimum angle $\theta_{s, \min }$ and the maximum angle $\theta_{s, \max }$ allowed to be subtended by the line segment $\left[S_{1} S_{2}\right]$ at $E$. More specifically, the following relation holds:

$$
\overline{C M}_{2}^{-1}=\theta_{s, \min }=180^{\circ}-\theta_{s, \max }
$$

From the inscribed circle theorem [14], for a given constant angle $\theta$, the contour of all the points at which the angle subtended by a line segment is either $\theta$ or $180^{\circ}-\theta$ is a pair of circles, the problematic region based on $C M_{2}$ can be easily determined in terms of $\overline{C M}_{2}^{-1}$ as shown in Fig. 3(b).

2) A Measure Based on Stochastic Properties of The Measurement Errors: Consider an arbitrary sensor pair - emitter $\left(S_{1}, S_{2}, E\right)$ setting as shown in Fig. 4, assuming without loss of generality that the position coordinates of the sensors are given by $S_{1}=[0,0]^{T}$ and $S_{2}=[0, d]^{T}$, where $d$ is an arbitrary positive number. Assume that the location of the emitter is estimated as the intersection $\hat{E}$ of the measured directions from $S_{1}$ and $S_{2}$, and the collinearity problem is defined as having the measured directions (rays) not intersecting at a unique point. We define a measure, $C M_{3}$, based on this collinearity problem definition and the stochastic properties of the DF measurement errors. Let $C M_{3}\left(S_{1}, S_{2}, E\right)$ be the probability

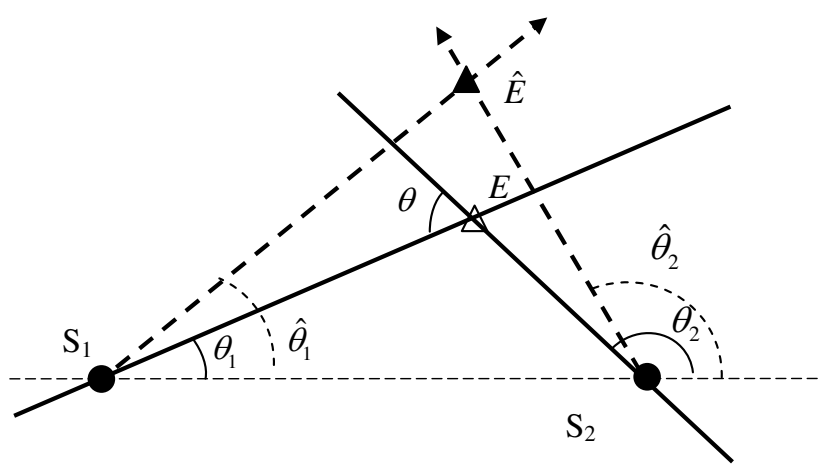

Fig. 4. Estimation of the emitter location by a pair of sensors as the intersection of measured directions (shown as dashed rays).

of encountering a collinearity problem for a given setting $\left(S_{1}, S_{2}, E\right)$ and the corresponding bearing angles $\theta_{1}, \theta_{2}$. First consider the case where the emitter $E$ lies above the line $S_{1} S_{2}$, i.e. $0^{\circ} \leq \theta_{1}, \theta_{2} \leq 180^{\circ}$. $\left(S_{1}, S_{2}, E\right)$ is free of the collinearity problem, as defined above, only in one of the two cases:

1) $0^{\circ} \leq \hat{\theta}_{1}<\hat{\theta}_{2}<180^{\circ}$

2) $0^{\circ} \geq \hat{\theta}_{1}>\hat{\theta}_{2}>-180^{\circ}$

Hence, we have

$$
C M_{3}\left(S_{1}, S_{2}, E\right)=1-p_{1}-p_{2}, \text { for } E=[x, y]^{T}, y \geq 0
$$


where $p_{1}=\operatorname{Pr}\left(0^{\circ} \leq \hat{\theta}_{1}<\hat{\theta}_{2}<180^{\circ}\right.$ and $p_{2}=\operatorname{Pr}\left(-180^{\circ} \leq\right.$ $\left.\hat{\theta}_{2}<\hat{\theta}_{1}<0^{\circ}\right)$. Following a set of similar steps or simply using a symmetry argument, for the case where the emitter $E$ lies below the line $S_{1} S_{2}$, we obtain

$$
\begin{array}{r}
C M_{3}\left(S_{1}, S_{2}, E\right)=\quad C M_{3}\left(S_{1}, S_{2},[x,-y]^{T}\right), \\
\text { for } E=[x, y]^{T}, y<0
\end{array}
$$

Next, we derive a more explicit expression for (5). Note that, for fixed values of $\theta_{1}, \theta_{2}$, we have

$$
f_{\hat{\theta}_{1}, \hat{\theta}_{2}}\left(\hat{\theta}_{1}, \hat{\theta}_{2}\right)=\quad f_{\tilde{\theta}_{1}, \tilde{\theta}_{2}}\left(\hat{\theta}_{1}-\theta_{1}, \hat{\theta}_{2}-\theta_{2}\right)
$$

Since by Assumption 1, $\tilde{\theta}_{1}$ and $\tilde{\theta}_{2}$ are uncorrelated jointly normal random variables with the joint density function

$$
f_{\tilde{\theta}_{1}, \tilde{\theta}_{2}}\left(\tilde{\theta}_{1}, \tilde{\theta}_{2}\right)=\quad \frac{1}{\sigma^{2}} g_{\sigma}\left(\tilde{\theta}_{1}\right) g_{\sigma}\left(\tilde{\theta}_{2}\right)
$$

where $g_{\sigma}(x) \triangleq g(x / \sigma)$ with $g(x)$ denoting the Gaussian function $\frac{1}{\sqrt{2 \pi}} e^{-x^{2} / 2}$, we have

$$
\begin{aligned}
& p_{1}=\frac{1}{\sigma^{2}} \int_{0}^{180} \int_{0}^{\hat{\theta}_{2}} g_{\sigma}\left(\hat{\theta}_{1}-\theta_{1}\right) g_{\sigma}\left(\hat{\theta}_{2}-\theta_{2}\right) d \hat{\theta}_{1} d \hat{\theta}_{2} \\
= & \frac{1}{\sigma} \int_{0}^{180} g_{\sigma}\left(\hat{\theta}_{2}-\theta_{2}\right)\left(G_{\sigma}\left(\hat{\theta}_{2}-\theta_{1}\right)-G_{\sigma}\left(-\theta_{1}\right)\right) d \hat{\theta}_{2}
\end{aligned}
$$

where $G_{\sigma}(x) \triangleq G(x / \sigma)$ with $G(x)$ denoting the cumulative Gaussian function $\int_{-\infty}^{x} g(\xi) d \xi$. Similarly, we obtain

$$
p_{2}=\frac{1}{\sigma} \int_{-180}^{0} g_{\sigma}\left(\hat{\theta}_{2}-\theta_{2}\right)\left(G_{\sigma}\left(-\theta_{1}\right)-G_{\sigma}\left(\hat{\theta}_{2}-\theta_{1}\right)\right) d \hat{\theta}_{2}
$$

From (7) and (8) we have, for $E=[x, y]^{T}, y \geq 0$,

$$
\begin{aligned}
& C M_{3}\left(S_{1}, S_{2}, E\right)= \\
& 1-\frac{1}{\sigma} \int_{-180}^{180} g_{\sigma}\left(\hat{\theta}_{2}-\theta_{2}\right)\left(G_{\sigma}\left(\hat{\theta}_{2}-\theta_{1}\right)-G_{\sigma}\left(-\theta_{1}\right)\right) \operatorname{sgn}\left(\hat{\theta}_{2}\right) d \hat{\theta}_{2}
\end{aligned}
$$

Fig. 5(a) depicts the region $\left\{E: C M_{3}\left(S_{1}, S_{2}, E\right) \geq \overline{C M}_{3}\right\}$ for $S_{1}=[0,0]^{T}(\mathrm{~km}), S_{2}=[1,0]^{T}(\mathrm{~km}), \overline{C M}_{3}=10^{-6}$.

3) A Jacobian-Based Measure: As a second approach to fine-tune $C M_{2}$, we define a mapping $f(\cdot)$ from the bearing angles $\theta_{1}, \theta_{2}$ of the sensors $S_{1}, S_{2}$ to the position coordinates $(x, y)$ of the emitter $E$, and using the Jacobian of $\boldsymbol{f}(\cdot)$, we analyze the sensitivity of the estimated coordinates of $E$ with respect to variations in the bearing angles measured by the sensors $S_{1}$ and $S_{2}$. The mapping $\boldsymbol{f}(\cdot)$ is given by

$$
E=\left[\begin{array}{l}
x \\
y
\end{array}\right]=\boldsymbol{f}\left(\left[\begin{array}{l}
\theta_{1} \\
\theta_{2}
\end{array}\right]\right)=d\left[\begin{array}{l}
\frac{\cos \theta_{1} \sin \theta_{2}}{\sin \left(\theta_{2}-\theta_{1}\right)} \\
\frac{\sin \theta_{1} \sin \theta_{2}}{\sin \left(\theta_{2}-\theta_{1}\right)}
\end{array}\right]
$$

The corresponding Jacobian is given by

$$
\begin{gathered}
J_{f}\left(\left[\begin{array}{l}
\theta_{1} \\
\theta_{2}
\end{array}\right]\right)=\left[\begin{array}{cc}
\frac{\partial x}{\partial \theta_{1}} & \frac{\partial x}{\partial \theta_{2}} \\
\frac{\partial y}{\partial \theta_{1}} & \frac{\partial y}{\partial \theta_{2}}
\end{array}\right] \\
=\frac{d}{1-\cos 2\left(\theta_{2}-\theta_{1}\right)}\left[\begin{array}{cc}
\sin 2 \theta_{2} & -\sin 2 \theta_{1} \\
1-\cos 2 \theta_{2} & \cos 2 \theta_{1}-1
\end{array}\right]
\end{gathered}
$$

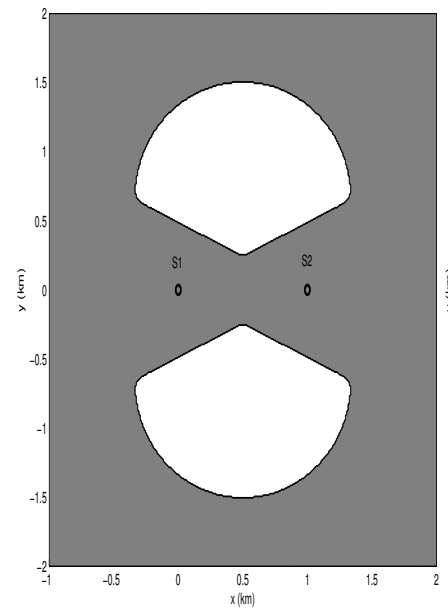

(a)

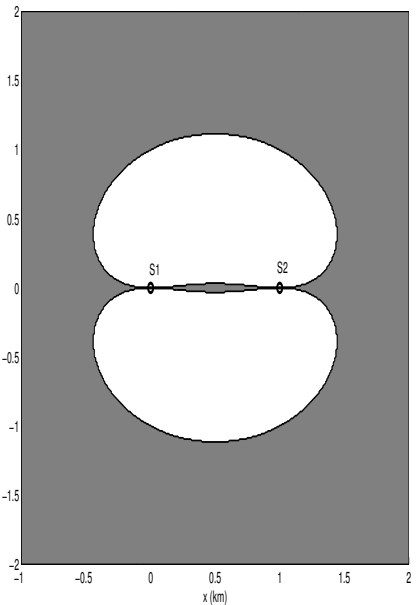

(b)
Fig. 5. Problematic regions (a) $\left\{E: C M_{3}\left(S_{1}, S_{2}, E\right) \geq \overline{C M}_{3}\right\}$ with $\overline{C M}_{3}=10^{-6}$ and (b) $\left\{E: C M_{4}\left(S_{1}, S_{2}, E\right) \geq \overline{C M}_{4}\right\}$ with $\overline{C M}_{4}=$ $2\left(\mathrm{~km}^{2}\right)$ for a sensor pair at $S_{1}=[0,0]^{T}(\mathrm{~km}), S_{2}=[1,0]^{T}(\mathrm{~km})$ : For an emitter located in the shaded area, collinearity problems are likely to happen.

Similar to Section III-C2, assume that the location of the emitter is estimated as the intersection $\hat{E}$ of the measured directions from $S_{1}$ and $S_{2}$, i.e.,

$$
\hat{E}=\left[\begin{array}{l}
\hat{x} \\
\hat{y}
\end{array}\right]=\boldsymbol{f}\left(\left[\begin{array}{l}
\hat{\theta}_{1} \\
\hat{\theta}_{2}
\end{array}\right]\right)
$$

Then the relation between the localization error $\tilde{E}=[\tilde{x}, \tilde{y}]^{T}=$ $\hat{E}-E$ and the DF measurement errors $\tilde{\theta}_{1}, \tilde{\theta}_{2}$ can be approximated by

$$
\tilde{E}=\left[\begin{array}{c}
\tilde{x} \\
\tilde{y}
\end{array}\right] \cong J_{f}\left(\left[\begin{array}{l}
\theta_{1} \\
\theta_{2}
\end{array}\right]\right)\left[\begin{array}{l}
\tilde{\theta}_{1} \\
\tilde{\theta}_{2}
\end{array}\right]
$$

Based on (13), we define our final $\mathrm{CM}, C M_{4}$ as follows:

$$
C M_{4}\left(S_{1}, S_{2}, E\right) \triangleq\left|\operatorname{det}\left(J_{f}\left(\left[\theta_{1}, \theta_{2}\right]^{T}\right)\right)\right|
$$

noting that a further measure along these lines would be provided by $C M_{4}^{\prime}\left(S_{1}, S_{2}, E\right) \triangleq \operatorname{trace}\left(J_{f}^{T} J_{f}\right)^{1 / 2}$. Fig. 5(b) depicts the problematic region $\left\{E: C M_{1}\left(S_{1}, S_{2}, E\right) \geq \overline{C M}_{1}\right\}$ for $S_{1}=[0,0]^{T}(\mathrm{~km}), S_{2}=[1,0]^{T}(\mathrm{~km}), \overline{C M}_{4}=2\left(\mathrm{~km}^{2}\right)$.

\section{Summary and Comparison}

In this section we have established four collinearity measures $C M_{1}$ to $C M_{4}$ for analyzing two sensors - one emitter settings with respect to potential geometric problems. Each of the four measures can be used to identify and quantify the potential collinearity problem from the aspect that is used in defining it, e.g. if the aim is to find and quantify the settings which suffer from large EDURs one may use $C M_{1}$, while $C M_{4}$ is a more suitable measure if the focus is sensitivity of a location estimator like (12) to direction measurement errors.

Figs. 3, 5, demonstrate that all the four measures hold some common properties such as symmetry with respect to the line $S_{1} S_{2}$ as well as the bisector of the line segment $\left[S_{1} S_{2}\right]$; having large values for the cases where the emitter $E$ is on the line $S_{1} S_{2}$ or distant from $S_{1}$ and $S_{2}$ (and hence identifying these 
cases as problematic ones); having small values for the case where the emitter $E S_{1} S_{2}$ is a equilateral triangle (and hence identifying this case as a non-problematic one).

In terms of computational complexity, $C M_{2}$ is the simplest to compute, and can be used as a simple measure in cases where fast computations and decisions are required. Considering the bases of definitions, $C M_{1}$ and $C M_{4}$ have more practical implications and can be found as more practical measures, especially in cases where there is no requirement of fast computations; noting that between $C M_{1}$ and $C M_{4}, C M_{4}$ is simpler to compute.

\section{Extension to Cases with More than Two SENSORS AND APPLICATIONS}

The four collinearity measures $C M_{1}$ to $C M_{4}$ established in Section III can also be used in analyzing the sensor-emitter settings involving a network of more than two sensors with respect to potential geometric problems. For such settings, considering only the cooperative localization between pairs of (neighbor) sensors, the problematic region for the whole sensor network can be defined as the intersection of the sensor-pairwise problematic regions, where the sensor-pair-wise problematic regions are determined as explained in Section III.

Based on the above definition, the problematic region for a three sensors - one emitter setting $\left(S_{1}, S_{2}, S_{3}, E\right)$ with $S_{1}=[0,0]^{T}(\mathrm{~km}), S_{2}=[1,0]^{T}(\mathrm{~km}), S_{3}=[0.5,0.87]^{T}$ $(\mathrm{km})$ is shown in Fig. 6 (a) for the case with $C M_{1}\left(\overline{C M}_{1}=\right.$ $\left.0.03\left(\mathrm{~km}^{2}\right)\right)$ and in Fig. 6 (b) for the case with $C M_{2}$.

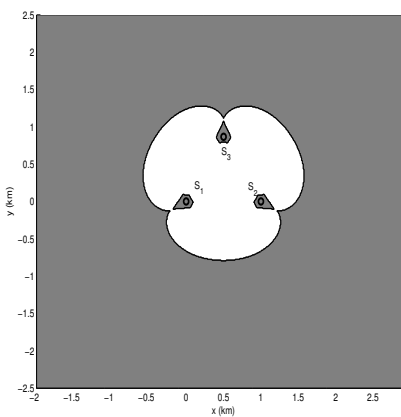

(a)

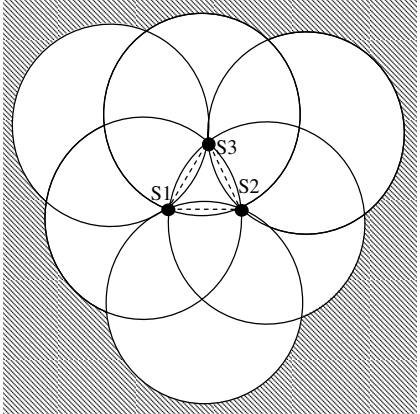

(b)
Fig. 6. Problematic region for a sensor triple at $\left(S_{1}, S_{2}, S_{3}, E\right)$ with $S_{1}=$ $[0,0]^{T}(\mathrm{~km}), S_{2}=[1,0]^{T}(\mathrm{~km}), S_{3}=[0.5,0.87]^{T}(\mathrm{~km})$ based on (a) $C M_{1}$ with $\sigma=5^{\circ}, \overline{C M}_{1}=0.03\left(\mathrm{~km}^{2}\right)$ and (b) based on $C M_{2}$.

Note that the above definition of problematic region based on any of the collinearity measures $C M_{1}$ to $C M_{4}$ can be used in sensor coverage problems to determine the optimal distribution of sensors in an area of interest in terms of robustness to potential geometric problems. A natural cost function in such a coverage problem would be the total area of problematic region for a pre-defined collinearity measure among $C M_{i}$ and a pre-determined threshold $\overline{C M}_{i}(i \in\{1,2,3,4\})$. Another potential application of the above problematic region concept is in determining the sub-networks of a sensor network the sensors in which cooperate in emitter localization.
Yet another application of the proposed collinearity measures and the problematic region concept is the avoidance of initialization of various iterative location estimators, including the MLE. First note that for a two sensors - one emitter setting, localizing the emitter from a pair of DF measurements, if bias issues are not considered, is simply a matter of determining where the two lines of bearing intersect. For three or more sensors and the corresponding bearing measurements, however, the concept of best estimate appears. From the MLE perspective, the best estimate for the emitter location is defined as the one that maximizes the likelihood function. For DF localization, in an $N$ sensors - one emitter $(N \geq 2)$ case, the likelihood function is

$$
\begin{aligned}
& l(\hat{E})=\quad-\epsilon^{T}(\hat{E}) \Sigma_{\theta}^{-1} \boldsymbol{\epsilon}(\hat{E}) \\
& \Sigma_{\theta}=\quad\left[\begin{array}{cccc}
\sigma_{1}^{2} & 0 & \cdots & 0 \\
0 & \sigma_{2}^{2} & \ddots & 0 \\
\vdots & \ddots & \ddots & \vdots \\
0 & \cdots & 0 & \sigma_{n}^{2}
\end{array}\right]
\end{aligned}
$$

where $\epsilon(\hat{E})=\left[\begin{array}{lll}\epsilon_{1}(\hat{E}) & \cdots & \epsilon_{N}(\hat{E})\end{array}\right]^{T}, \quad \epsilon_{i}(\hat{E})=\hat{\theta}_{i}-$ $\tan ^{-1}\left(\frac{\hat{y}-y_{i}}{\hat{x}-x_{i}}\right), i=1, \cdots, N, \tan ^{-1}\left(\left(\hat{y}-y_{i}\right) /\left(\hat{x}-x_{i}\right)\right)$ is the inverse tangent of $\left(\hat{y}-y_{i}\right) /\left(\hat{x}-x_{i}\right)$ with the signs of both $\hat{x}-x_{i}$ and $\hat{y}-y_{i}$ being used to determine the correct quadrant of the result, and $\sigma_{i}^{2}$ denotes the variance of bearing measurements from the sensor $S_{i}$.

Note that for the two sensors - one emitter $(N=2)$ setting considered in Section III-C3 and the mapping (12), $\epsilon(\hat{E})=0$ and hence (12) is a minimizer of (15) and hence an MLE. For $N>2$, the maximum likelihood estimate is usually obtained starting with an initial estimate and iterating the estimate aiming to reach a point where

$$
\partial l(\hat{E}) / \partial \hat{E}=0
$$

One common method for solving (16) iteratively is the TaylorSeries method also known as the Gauss-Newton method as outlined in [3]. However in problematic geometry cases, which may potentially be identified using $C M_{4}$ presented in Section III-C3 and the collinearity measure extensions to cases for $N>3$ discussed in this section, the Taylor series method may not converge to a solution unless the initial estimate is very close to the true emitter location and the step-size is chosen appropriately [15].

\section{CONCluding REMARKS}

In this paper we have analyzed sensor-emitter placements that cause large biases or the failure of estimates to converge to a unique solution in cooperative DF-based localization. These problematic placements are characterized by near collinearity. We have proposed four methods for measuring nearcollinearity in arbitrary two sensors - one emitter configurations which enable quick and easy identification of cases in which DF localization will fail to obtain a solution or give unreliable results. We have informally discussed application of these methods in analyzing configurations with more than 
two sensors, selecting the positions of sensors in a cooperative DF-based localization network for optimal coverage robust to potential geometric problems, optimal teaming of sensor nodes for cooperative localization, and elimination of ill conditioned initialization of iterative localization algorithms. Formal treatment of each application is a future research topic.

\section{ACKNOWLEDGMENT}

NICTA is funded by the Australian Government as represented by the Department of Broadband, Communications and the Digital Economy and the Australian Research Council through the ICT Centre of Excellence Program.

\section{REFERENCES}

[1] D. Adamy, EW 101 : A First Course in Electronic Warfare. Boston: Artech House, 2001.

[2] C. Ancker, "Airborne direction finding-The theory of navigation errors," IRE Trans. Aero. and Navig. Electronics, vol. 5, pp. 199 - 210, 1958.

[3] D. J. Torrieri, "Statistical-theory of passive location systems," IEEE Trans. Aero. and Electronic Systems, vol. 20, no. 2, pp. 183-198, 1984.

[4] M. Gavish and A. J. Weiss, "Performance analysis of bearing-only target location algorithms," IEEE Trans. on Aerospace and Electronic Systems, vol. 28, no. 3, pp. 817-828, 1992.

[5] D. Koks, "Numerical calculations for passive geolocation scenarios," Australian Defence Science and Technology Organisation, Edinburgh, SA, Australia, Tech. Rep. DSTO-RR-0319, May 2007.

[6] R. G. Stansfield, "Statistical theory of DF fixing," Journal of IEE, vol. 14, no. III A, 15, pp. 762-770, 1947.

[7] H. E. Daniels, "The theory of position finding," Journal of Royal Statistical Society, vol. 13, no. 2, 1951.

[8] S. Nardone, A. Lindgren, and K. Gong, "Fundamental properties and performance of conventional bearings-only target motion analysis," IEEE Trans. Automatic Control, vol. 29, no. 9, pp. 775-787, 1984.

[9] K. Doğançay, "On the bias of linear least squares algorithms for passive target localization,” Signal Processing, vol. 84, no. 3, pp. 475-486, 2004.

[10] M. S. Kay, Fundamentals of Statistical Signal Processing: Estimation Theory. Prentice-Hall, 1993.

[11] A. Bishop, B. Fidan, B. Anderson, K. Doğançay, and P. Pathirana, "Optimality analysis of sensor-target geometries in passive localization: Part 1 - bearing-only localization," in Proc. 3rd Int. Conf. on Intelligent Sensors, Sensor Networks and Information Processing (ISSNIP), December 2007, pp. 7-12.

[12] K. Doğançay and H. Hmam, "Optimal angular sensor separation for aoa localization," Signal Processing, vol. 88, pp. 1248-1260, May 2008.

[13] Y. Oshman and P. Davidson, "Optimization of observer trajectories for bearings-only target localization," IEEE Trans. on Aerospace and Electronic Systems, vol. 35, no. 3, pp. 892-902, 1999.

[14] C. Ogilvy, Excursions in Geometry. Oxford University Press, 1969.

[15] J. P. Le Cadre and C. Jauffret, "On the convergence of iterative methods for bearings-only tracking," IEEE Trans. on Aerospace and Electronic Systems, vol. 35, no. 3, pp. 801-818, 1999. 